MATHEMATICS OF COMPUTATION

Volume 68, Number 227, Pages 1079-1095

S 0025-5718(99)01073-X

Article electronically published on February 8, 1999

\title{
THE PIECEWISE POLYNOMIAL COLLOCATION METHOD FOR NONLINEAR WEAKLY SINGULAR VOLTERRA EQUATIONS
}

\author{
HERMANN BRUNNER, ARVET PEDAS, AND GENNADI VAINIKKO
}

\begin{abstract}
Second-kind Volterra integral equations with weakly singular kernels typically have solutions which are nonsmooth near the initial point of the interval of integration. Using an adaptation of the analysis originally developed for nonlinear weakly singular Fredholm integral equations, we present a complete discussion of the optimal (global and local) order of convergence of piecewise polynomial collocation methods on graded grids for nonlinear Volterra integral equations with algebraic or logarithmic singularities in their kernels.
\end{abstract}

\section{INTRODUCTION}

The solution of a second-kind Fredholm integral equation with weakly singular kernel is typically nonsmooth near the boundary of the domain of integration (its derivatives are unbounded). We refer to Richter [28], Pedas [21], [22], Schneider [30], Pitkäranta [24], [25], Vainikko and Pedas [41], Graham [8], Vainikko, Pedas and Uba [42], Vainikko [39], [40], Uba [34], Kaneko, Noren and Xu [12], R. Kangro [14], U. Kangro [15], [16], Pedas and Vainikko [23].

If one wants to obtain a high-order convergence in a numerical method for these equations one has to take into account, in some way, the singular behaviour of the exact solution. This can be done by using polynomial splines on special graded grids. The theory of graded grids in the approximation by polynomial splines goes back to Rice [27] (for a complete theory see, for example, de Boor [1] or Schumaker [32]. In the numerical solution of second-kind Fredholm integral equations with weakly singular kernels, graded grids were used by Chandler [7], Schneider [31], Vainikko and Uba [43], Graham [9], Vainikko, Pedas and Uba [42], Vainikko [37], [38], [40], Hackbusch [10], Uba [35], [36], Kaneko, Noren and Xu [13], Tamme [33], Pedas and Vainikko [24].

A similar situation arises in the case of Volterra integral equations. For secondkind Volterra equations with weakly singular kernels the nonsmooth behaviour of solutions occurs near the initial point of integration. We refer to Miller and Feldstein [20], de Hoog and Weiss [11], Logan [18], Lubich [19], Brunner [2], [4], Brunner and van der Houwen [6]. Again, if one is interested in finding an approximate solution which exhibits high-order accuracy, one may resort to approximation with polynomial splines on graded grids which reflect the singular behaviour of the exact

Received by the editor September 2, 1997.

1991 Mathematics Subject Classification. Primary 65R20, 45E10, 45B05.

Key words and phrases. Nonlinear weakly singular Volterra and Fredholm integral equations, piecewise polynomial collocation, graded grids, optimal order of convergence.

(C)1999 American Mathematical Society 
solution near the initial point. In the numerical solution of second-kind Volterra integral equations with weakly singular kernels graded grids were used by Brunner [4], [3], Brunner and van der Houwen [6], and Brunner [5].

In the previous papers the case of Fredholm equations was considered independently of Volterra equations and, vice versa, the results for Volterra equations were obtained independently of existing results on Fredholm equations.

The purpose of the present paper is to show how in the analysis of the numerical solution of weakly singular Volterra integral equations by polynomial splines on graded grids it is possible to use corresponding existing results for Fredholm equations. More precisely, on the basis of results from [40, pp. 22-23] we shall study the smoothness of solutions and the piecewise polynomial collocation method for a rather wide class of nonlinear weakly singular Volterra integral equations. Using special collocation points in conjunction with graded grids, we derive global convergence estimates and analyze a superconvergence effect at the collocation points. The main results of the paper considerably extend known ones and are formulated in Theorems 2.1-2.4 (see Section 2). The proofs of these assertions are given in Section 4 and are based on the following simple idea: for a given Volterra equation

$$
u(t)=\int_{0}^{t} K(t, s, u(s)) d s+f(t), \quad 0 \leq t \leq T
$$

we find the appropriate extensions $\tilde{K}(t, s, u)$ and $\tilde{f}(t)$ for $K(t, s, u)$ and $f(t)$ so that from the corresponding results of Section 3 for the Fredholm equation

$$
u(t)=\int_{0}^{2 T} \tilde{K}(t, s, u(s)) d s+\tilde{f}(t), \quad 0 \leq t \leq 2 T,
$$

we can derive all results formulated in Theorems 2.1-2.4 for the Volterra equation (1.1). Finally, in Section 5 we present some numerical illustrations.

\section{VOLTERRA EQUATIONS WITH WEAKLY SINGULAR KERNELS}

2.1. Smoothness of the solution. Consider the nonlinear Volterra equation

$$
u(t)=\int_{0}^{t} K(t, s, u(s)) d s+f(t), \quad 0 \leq t \leq T
$$

The following assumptions (V1)-(V3) are made.

(V1) The kernel $K=K(t, s, u)$ is $m$ times $(m \geq 1)$ continuously differentiable with respect to $t, s, u$ for $t \in[0, T], s \in[0, t), u \in \mathbb{R}$, and there exists a real number $\nu \in(-\infty, 1)$ such that for $0 \leq s<t \leq T, u \in \mathbb{R}$, and for nonnegative integers $i, j, k$ with $i+j+k \leq m$, the following inequalities hold:

$$
\left|\left(\frac{\partial}{\partial t}\right)^{i}\left(\frac{\partial}{\partial t}+\frac{\partial}{\partial s}\right)^{j}\left(\frac{\partial}{\partial u}\right)^{k} K(t, s, u)\right| \leq b_{1}(|u|) \begin{cases}1 & \text { if } \nu+i<0 \\ 1+|\log | t-s|| & \text { if } \nu+i=0 \\ |t-s|^{-\nu-i} & \text { if } \nu+i>0\end{cases}
$$


and

$$
\begin{gathered}
\left|\left(\frac{\partial}{\partial t}\right)^{i}\left(\frac{\partial}{\partial t}+\frac{\partial}{\partial s}\right)^{j}\left(\frac{\partial}{\partial u}\right)^{k} K\left(t, s, u_{1}\right)-\left(\frac{\partial}{\partial t}\right)^{i}\left(\frac{\partial}{\partial t}+\frac{\partial}{\partial s}\right)^{j}\left(\frac{\partial}{\partial u}\right)^{k} K\left(t, s, u_{2}\right)\right| \\
\quad \leq b_{2}\left(\operatorname { m a x } \{ | u _ { 1 } | , | u _ { 2 } | \} | u _ { 1 } - u _ { 2 } | \left\{\begin{array}{ll}
1 & \text { if } \nu+i<0, \\
1+|\log | t-s|| & \text { if } \nu+i=0, \\
|t-s|^{-\nu-i} & \text { if } \nu+i>0,
\end{array}\right.\right.
\end{gathered}
$$

where the functions $b_{1}:[0, \infty) \rightarrow[0, \infty)$ and $b_{2}:[0, \infty) \rightarrow[0, \infty)$ are assumed to be monotonically increasing.

(V2) $f \in C^{m, \nu}(0, T]$, i.e. $f(t)$ is $m$ times continuously differentiable for $0<t \leq T$ and the estimate

$$
\left|f^{(k)}(t)\right| \leq \operatorname{const}_{f} \begin{cases}1 & \text { if } k<1-\nu \\ 1+|\log t| & \text { if } k=1-\nu, \quad t \in(0, T], \\ t^{1-\nu-k} & \text { if } k>1-\nu,\end{cases}
$$

holds for $k=0,1, \ldots, m$.

(V3) The integral equation (1.1) has a solution $u_{0} \in L^{\infty}(0, T)$.

Notice that conditions (V1) and (V2) guarantee the existence and uniqueness of the solution to $(2.1)$ on some interval $\left[0, T_{0}\right], T_{0} \leq T$. On $[0, T]$ the existence and uniqueness of the solution will be guaranteed if we impose the following global Lipschitz condition on $K(t, s, u)$ : for $0 \leq t \leq T, 0 \leq s<t, u_{1}, u_{2} \in \mathbb{R}$, there holds

$$
\left|K\left(t, s, u_{1}\right)-K\left(t, s, u_{2}\right)\right| \leq b\left|u_{1}-u_{2}\right| \begin{cases}1 & \text { if } \quad \nu<0 \\ 1+|\log | t-s|| & \text { if } \quad \nu=0 \\ |t-s|^{-\nu} & \text { if } \quad \nu>0\end{cases}
$$

with a constant $b$ independent of $u_{1}$ and $u_{2}$ (cf. (2.3), $i=j=k=0$ ). We do not restrict the problem by this global Lipschitz condition, but assume (V3). Moreover, assuming (V3) we in fact could replace (V1) by the corresponding local condition for $t \in[0, T], s \in[0, t),\left|u-u_{0}(s)\right| \leq \delta$ with some $\delta>0$. Outside this set, the kernel $K(t, s, u)$ is involved neither in (2.1) nor in the corresponding collocation method described in Section 2.2. Nevertheless, we remain with the formulation of (V1) given above.

It follows from (V1) that the kernel $K(t, s, u)$ may possess a weak singularity as $s \rightarrow t(i=j=k=0,0 \leq \nu<1)$. In the case $\nu<0$, the kernel $K(t, s, u)$ is bounded for $0 \leq s \leq t \leq T$ and fixed $u \in \mathbb{R}$, but its derivatives may be singular as $s \rightarrow t$. Often the kernel $K$ has the form

$$
K(t, s, u)=a(t, s, u)(t-s)^{-\beta}, \quad \beta<1,
$$

or

$$
K(t, s, u)=a(t, s, u) \log (t-s),
$$

where $a(t, s, u)$ is an $m$-smooth function of its arguments for $0 \leq s \leq t \leq T, u \in \mathbb{R}$. Clearly, condition (V1) is satisfied in these examples with $\nu=\beta$ and $\nu=0$, respectively. Actually, (V1) remains fulfilled even if derivatives of $a(t, s, u)$ have certain (sufficiently weak) singularities as $s \rightarrow t$.

The following result states the regularity properties of solutions of equation (2.1). 
Theorem 2.1. Let the conditions (V1) and (V2) be fulfilled. If equation (2.1) has a solution $u$ in $L^{\infty}(0, T)$, then $u \in C^{m, \nu}(0, T]$.

The proof of Theorem 2.1 is given in Section 4. We remark that smoothness properties of solutions to (more special) weakly singular Volterra equations are analyzed in $[19$, p. 6$]$.

We note also that $u \in C^{m, \nu}(0, T]$ having an integrable derivative in $(0, T]$ can be extended up to a continuous function on $[0, T]$. The extended function will be denoted again by $u$.

2.2. Piecewise polynomial approximation of the solution. For given $N \in \mathbb{N}$ let $0=t_{0}<t_{1}<\ldots<t_{N}=T$ be a partition of the interval $[0, T]$ with grid points

$$
t_{j} \equiv t_{j}^{(N)}=(j / N)^{r} T, \quad j=0,1, \ldots, N,
$$

where $r \in \mathbb{R}, r \geq 1$. For $r=1$, the grid points $t_{0}, t_{1}, \ldots, t_{N}$ are distributed uniformly; for $r>1$, the grid points are more densely clustered near the left endpoint of the interval $[0, T]$. In every subinterval $\left[t_{j-1}, t_{j}\right](j=1, \ldots, N)$ we choose $m$ collocation points

$$
\xi_{j i}=t_{j}+\frac{\eta_{i}+1}{2}\left(t_{j+1}-t_{j}\right), i=1, \ldots, m,
$$

where $\eta_{1}, \ldots, \eta_{m}$ do not depend on $N$ and satisfy

$$
-1 \leq \eta_{1}<\eta_{2}<\ldots<\eta_{m} \leq 1
$$

To a continuous function $u:[0, T] \rightarrow \mathbb{R}$ we assign a piecewise polynomial interpolant $P_{N} u:[0, T] \rightarrow \mathbb{R}$ as follows: 1$)$ on every subinterval $\left[t_{j-1}, t_{j}\right](j=$ $1, \ldots, N), P_{N} u$ is a polynomial of degree $m-1$; 2) $P_{N} u$ interpolates $u$ at the points $\xi_{j 1}, \ldots, \xi_{j m}$ :

$$
\left(P_{N} u\right)\left(\xi_{j i}\right)=u\left(\xi_{j i}\right), i=1, \ldots, m ; j=1, \ldots, N .
$$

Thus, the interpolation function $\left(P_{N} u\right)(t)$ is independently defined in every subinterval $\left[t_{j-1}, t_{j}\right](j=1, \ldots, N)$ and may be discontinuous at the interior grid points $t=t_{j}(j=1, \ldots, N-1)$. We may treat $P_{N} u$ as a two-valued function at these points. Note that in the case $\eta_{1}=-1$ and $\eta_{m}=1, P_{N} u$ is a continuous function on $[0, T]$.

Let $E_{N}$ denote the range of the interpolatory projection $P_{N}$, that is, the set of all piecewise polynomial functions on $[0, T]$ which are polynomials of degree not exceeding $m-1$ on every subinterval $\left[t_{j-1}, t_{j}\right](j=1, \ldots, N)$. We introduce also the notation

$$
h=T / N .
$$

We look for an approximate solution $u_{N} \in E_{N}$ to the integral equation (2.1) which satisfies this equation at the collocation points (2.6):

$$
\left[u_{N}(t)-\int_{0}^{t} K\left(t, s, u_{N}(s)\right) d s-f(t)\right]_{t=\xi_{j i}}=0, \quad i=1, \ldots, m ; j=1, \ldots, N .
$$

Conditions (2.8) form a system of equations whose exact form is determined by the choice of a basis of $E_{N}$. For instance, in the interval $\left[t_{j-1}, t_{j}\right](j=1, \ldots, N)$ 
we may use the representation

$$
u_{N}(s)=\sum_{i=1}^{m} c_{j i} \varphi_{j i}(s), \quad s \in\left[t_{j-1}, t_{j}\right]
$$

where $\varphi_{j i}(s)\left(s \in\left[t_{j-1}, t_{j}\right]\right)$ is the polynomial of degree $m-1$ such that

$$
\varphi_{j i}\left(\xi_{j k}\right)=\left\{\begin{array}{ll}
1 & \text { if } k=i, \\
0 & \text { if } k \neq i,
\end{array} \quad k=1, \ldots, m\right.
$$

The collocation conditions (2.8) then lead to the following (nonlinear) system of algebraic equations for the coefficients $\left\{c_{j i}\right\}$ :

$$
\begin{gathered}
c_{j i}=f\left(\xi_{j i}\right)+\sum_{l=1}^{j-1} \int_{t_{l-1}}^{t_{l}} K\left(\xi_{j i}, s, \sum_{k=1}^{m} c_{l k} \varphi_{l k}(s)\right) d s \\
+\int_{t_{j-1}}^{\xi_{j i}} K\left(\xi_{j i}, s, \sum_{k=1}^{m} c_{j k} \varphi_{j k}(s)\right) d s, i=1, \ldots, m ; j=1, \ldots, N .
\end{gathered}
$$

This represents a recursive process: First, the coefficients $c_{11}, \ldots, c_{1 m}$ can be found by solving the system

$$
c_{1 i}=f\left(\xi_{1 i}\right)+\int_{0}^{\xi_{1 i}} K\left(\xi_{1 i}, s, \sum_{k=1}^{m} c_{1 k} \varphi_{1 k}(s)\right) d s, i=1, \ldots, m .
$$

Using $c_{11}, \ldots, c_{1 m}$, one can then find $c_{21}, \ldots, c_{2 m}$ from the system

$$
\begin{aligned}
& c_{2 i}=f\left(\xi_{2 i}\right)+\int_{0}^{t_{1}} K\left(\xi_{2 i}, s, \sum_{k=1}^{m} c_{1 k} \varphi_{1 k}(s)\right) d s \\
& +\int_{t_{1}}^{\xi_{2 i}} K\left(\xi_{2 i}, s, \sum_{k=1}^{m} c_{2 k} \varphi_{2 k}(s)\right) d s, i=1, \ldots, m .
\end{aligned}
$$

Generally, using $c_{11}, \ldots, c_{1 m}, \ldots, c_{j-1,1}, \ldots, c_{j-1, m}$, the coefficients $c_{j 1}, \ldots, c_{j m}$ can be found from the $m$ equations (2.10) with corresponding $j$. Thus at every step (on every subinterval $\left[t_{j-1}, t_{j}\right]$ ) one has to solve a system of $m$ nonlinear equations; the initial guess can be chosen by using the solution on the previous subinterval. For the first block system (2.11) a suitable initial guess is $c_{1 i}=f\left(\xi_{1 i}\right), i=1, \ldots, m$.

The following theorem states the global convergence rate for the collocation method (2.8).

Theorem 2.2. Let assumptions (V1)-(V3) be fulfilled and let the collocation points (2.6) corresponding to the gridpoints (2.5) be used.

Then there exist an $N_{0} \in \mathbb{N}$ and a real number $\delta_{0}>0$ such that, for $N>N_{0}$, the collocation method (2.8) defines a unique approximation $u_{N} \in E_{N}$ to the solution $u_{0}$ of equation (2.1) satisfying $\left\|u_{N}-u_{0}\right\|_{L^{\infty}(0, T)} \leq \delta_{0}$. The following error estimates hold:

1) if $m<1-\nu$ then

$$
\max _{0 \leq t \leq T}\left|u_{N}(t)-u_{0}(t)\right| \leq \operatorname{ch}^{m} \text { for } r \geq 1
$$


2) if $m=1-\nu$ then

$$
\max _{0 \leq t \leq T}\left|u_{N}(t)-u_{0}(t)\right| \leq c \begin{cases}h^{m}(1+|\log h|) & \text { for } r=1, \\ h^{m} & \text { for } r>1,\end{cases}
$$

and

$$
\left\|u_{N}-u_{0}\right\|_{L^{p}(0, T)} \leq \operatorname{ch}^{m} \text { for } r \geq 1,1 \leq p<\infty ;
$$

3) if $m>1-\nu$ then

$$
\max _{0 \leq t \leq T}\left|u_{N}(t)-u_{0}(t)\right| \leq c\left\{\begin{array}{llr}
h^{r(1-\nu)} & \text { for } 1 \leq r \leq \frac{m}{1-\nu} \\
h^{m} & \text { for } \quad r \geq \frac{m}{1-\nu}
\end{array}\right.
$$

and for $1 \leq p<\infty$,

$$
\left\|u_{N}-u_{0}\right\|_{L^{p}(0, T)} \leq c \begin{cases}h^{r\left(1-\nu+\frac{1}{p}\right)} & \text { if } 1 \leq r<\frac{m}{1-\nu+\frac{1}{p}}, m>1-\nu+\frac{1}{p}, \\ h^{m}(1+|\log h|)^{\frac{1}{p}} & \text { if } \quad r=\frac{m}{1-\nu+\frac{1}{p}}, m \geq 1-\nu+\frac{1}{p}, \\ h^{m} & \text { if } \quad r>\frac{m}{1-\nu+\frac{1}{p}}, r \geq 1\end{cases}
$$

4) if $r=r(m, \nu) \geq 1$ is restricted by conditions

$$
\begin{array}{ll}
r>\frac{m}{2(1-\nu)} & \text { for } 0 \leq \nu<1, \\
r>\frac{m}{2-\nu} & \text { for } \nu<0, \\
r \geq 1 & \text { for } \nu<0, \quad \nu<-(m-2),
\end{array}
$$

then

$$
\varepsilon_{N} \leq c h^{m}
$$

where

$$
\varepsilon_{N}=\max _{i=1, \ldots, m ; j=1, \ldots, N}\left|u_{N}\left(\xi_{j i}\right)-u_{0}\left(\xi_{j i}\right)\right|
$$

is the maximal error of the approximate solution $u_{N} \in E_{N}$ at the collocation points (2.6). The constants $c$ in (2.12)-(2.18) are independent of $h=T / N$.

The proof of Theorem 2.2 is given in Section 4 .

2.3. Superconvergence at the collocation points. We now assume that the points $\eta_{1}, \ldots, \eta_{m}$ in (2.6) (the points $\left.(2.7)\right)$ are the knots of the quadrature formula

$$
\int_{-1}^{1} \varphi(\xi) d \xi \approx \sum_{i=1}^{m} w_{i} \varphi\left(\eta_{i}\right),-1 \leq \eta_{1}<\ldots<\eta_{m} \leq 1,
$$

which is exact for polynomials of degree $m+\mu, \mu \in \mathbf{Z}, 0 \leq \mu \leq m-1$. Actually, the weights $w_{1}, \ldots, w_{m}$ of the quadrature formula (2.20) will not be used in our analysis. The case $\mu=m-1$ corresponds to the Gauss quadrature formula and is of the greatest interest in the following theorem.

Theorem 2.3. Let the following conditions be fulfilled:

$\left(\mathrm{V}^{\prime}\right)$ The kernel $K(t, s, u)$ and $\partial K(t, s, u) / \partial u$ are $m+\mu+1$ times $(m, \mu \in \mathbf{Z}$, $m \geq 1,0 \leq \mu \leq m-1)$ continuously differentiable with respect to $t, s, u$ for $t \in[0, T], s \in[0, t), u \in \mathbb{R}$, and satisfy (2.2) and (2.3) with $i+j+k \leq$ $m+\mu+1, \nu \in(-\infty, 1)$. 
$\left(\mathrm{V} 2^{\prime}\right) f \in C^{m+\mu+1, \nu}(0, T]$.

(V3) The integral equation (2.1) has a solution $u_{0} \in L^{\infty}(0, T)$.

(V4) The collocation points (2.6) are generated by the knots (2.7) of a quadrature formula (2.20) which is exact for all polynomials of degree $m+\mu, 0 \leq \mu \leq$ $m-1$.

(V5) The scaling parameter $r=r(m, \nu, \mu) \geq 1$ is subject to the restrictions

$$
\begin{array}{ll}
r>\frac{m}{1-\nu}, r \geq \frac{m+1-\nu}{2-\nu} & \text { if } 1-\nu<\mu+1, \\
r>\frac{m}{1-\nu}, r>\frac{m+\mu+1}{2-\nu} & \text { if } \mu+1 \leq 1-\nu<m, \\
r \geq \frac{m+\mu+1}{2-\nu}, r>1 & \text { if } 1-\nu=m, \\
r \geq \frac{m+\mu+1}{2-\nu} & \text { if } 1-\nu>m .
\end{array}
$$

Then

$$
\varepsilon_{N} \leq c h^{m} \begin{cases}h & \text { if } \nu<0, \\ h(1+|\log h|) & \text { if } \nu=0, \\ h^{1-\nu} & \text { if } \nu>0,\end{cases}
$$

where $\varepsilon_{N}$ is defined in (2.19) and $c$ is a positive constant which is independent of $h=T / N$.

The proof of Theorem 2.3 is given in Section 4 .

Theorem 2.4. Let the conditions of Theorem 2.3 hold. Assume additionally that:

(V6) $\nu<0, \mu \geq 1$, and for $0 \leq j \leq \min \{\mu-1,-\nu\}, 0 \leq k \leq \min \{\mu-1,-\nu\}$, the derivatives

$$
\left(\frac{\partial}{\partial s}\right)^{j}\left(\frac{\partial}{\partial u}\right)^{k+1} K(t, s, u)
$$

are bounded and continuous for $0 \leq t \leq T, 0 \leq s \leq t,|u| \leq \tau$ with any $\tau>0$, and $\left(\frac{\partial}{\partial s}\right)^{j}\left(\frac{\partial}{\partial u}\right)^{k+1} K(t, s, u) \rightarrow 0$ as $s \rightarrow t-0$.

Then

$$
\varepsilon_{N} \leq c h^{m} \begin{cases}h^{\mu+1} & \text { if } 1-\nu>\mu+1, \\ h^{\mu+1}(1+|\log h|) & \text { if } 1-\nu=\mu+1, \\ h^{1-\nu} & \text { if } 1-\nu<\mu+1,\end{cases}
$$

where $\varepsilon_{N}$ is defined in (2.19) and the constant $c$ is independent of $h=T / N$.

The proof of Theorem 2.4 is given in Section 4 .

Remark 2.1 (comment on the additional condition (V6)). Assumption (V1') of Theorem 2.3 guarantees the boundedness and continuity of the derivatives (2.23) for $j<\min \{\mu-1,-\nu\}, j+k \leq m+\mu+1$, on any set $0 \leq s<t \leq T,-\tau<u<\tau, \tau>0$; for $j=-\nu$ with $\nu \in \mathbf{Z},-\nu<\mu-1$, a logarithmic singularity may occur. Condition (V6) bans this possible singularity.

Remark 2.2. The estimate $\max _{0 \leq t \leq T}\left|u_{N}(t)-u_{0}(t)\right| \leq c h^{m}$ is of optimal order even for a function $u \in C^{\infty}[0, T]$. Theorem 2.2 shows that, using sufficiently great values of the scaling parameters $r$, the optimal accuracy $O\left(h^{m}\right)$ can be achieved for the collocation method (2.8). Theorems 2.3 and 2.4 show that superconvergence occurs at the collocation points. 
Remark 2.3. Under the conditions of Theorem 2.3

$$
\max _{0 \leq t \leq T}\left|\tilde{u}_{N}(t)-u_{0}(t)\right| \leq c h^{m} \begin{cases}h & \text { if } \nu<0, \\ h(1+|\log h|) & \text { if } \nu=0, \\ h^{1-\nu} & \text { if } \nu>0\end{cases}
$$

where

$$
\tilde{u}_{N}(t)=\int_{0}^{t} K\left(t, s, u_{N}(s)\right) d s+f(t)
$$

Under the conditions of Theorem 2.4,

$$
\max _{0 \leq t \leq T}\left|\tilde{u}_{N}(t)-u_{0}(t)\right| \leq c h^{m} \begin{cases}h^{\mu+1} & \text { if } 1-\nu>\mu+1 \\ h^{\mu+1}(1+|\log h|) & \text { if } 1-\nu=\mu+1 \\ h^{1-\nu} & \text { if } 1-\nu<\mu+1\end{cases}
$$

\section{Fredholm equations With WeAKLy Singular KeRnels}

In this section we formulate some results from [39, pp. 22-23] about Fredholm integral equations which give a basis for the proofs of the results presented in Section 2 .

Consider the nonlinear Fredholm integral equation

$$
u(t)=\int_{0}^{2 T} K(t, s, u(s)) d s+f(t), \quad 0 \leq t \leq 2 T .
$$

We make the following assumptions (F1) and (F2).

(F1) The kernel $K=K(t, s, u)$ is $m$ times $(m \geq 1)$ continuously differentiable with respect to $t, s, u$ for $t, s \in[0,2 T], t \neq s, u \in \mathbb{R}$, and there exists a real number $\nu \in(-\infty, 1)$ such that for nonnegative integers $i, j, k$ with $i+j+k \leq m$ inequalites (2.2) and (2.3) hold.

(F2) $f \in C^{m, \nu}(0,2 T)$, i.e. $f(t)$ is $m$ times continuously differentiable for $0<t<$ $2 T$, and the estimate

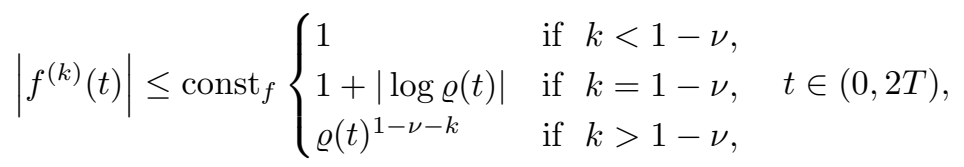

holds for $k=0,1, \ldots, m$, where $\varrho(t)=\min \{t, 2 T-t\}$.

Theorem 3.1 ([23], [39, p. 137]). Let conditions (F1) and (F2) be fulfilled. If the integral equation (3.1) has a solution $u \in L^{\infty}(0,2 T)$, then $u \in C^{m, \nu}(0,2 T)$.

In the sequel we shall assume that:

(F3) The integral equation (3.1) has a solution $u_{0} \in L^{\infty}(0,2 T)$ and the linearized equation

$$
v(t)=\int_{0}^{2 T} K_{0}(t, s) v(s) d s, \quad K_{0}(t, s)=\partial K(t, s, u) /\left.\partial u\right|_{u=u_{0}(s)},
$$

has in $L^{\infty}(0,2 T)$ only the trivial solution $v \equiv 0$. 
For given $N \subset \mathbb{N}$, let $0=t_{0}<t_{1}<\ldots<t_{2 N}=2 T$ be a partition of the interval $[0,2 T]$ with gridpoints $(2.5)$ and gridpoints $t_{N+1}, \ldots, t_{2 N}$ which are obtained by reflecting $t_{N-1}, \ldots, t_{0}$ with respect to $t_{N}=T$ :

$$
t_{j}=(j / N)^{r} T, \quad j=0,1, \ldots, N ; \quad t_{j+N}=2 T-t_{N-j}, \quad j=1, \ldots, N .
$$

In every subinterval $\left[t_{j-1}, t_{j}\right](j=1, \ldots, 2 N)$ we choose $m$ collocation points $(2.6)$ determined by the points $(2.7)$. We denote by $\tilde{E}_{N}$ the set of all piecewise polynomial functions on $[0,2 T]$ which are polynomials of degree not exceeding $m-1$ on every interval $\left[t_{j-1}, t_{j}\right](j=1, \ldots, 2 N)$. We look for an approximate solution $u_{N} \in \tilde{E}_{N}$ to the integral equation (3.1) which satisfies this equation at the collocation points (2.6) corresponding to the gridpoints (3.4):

$$
\begin{gathered}
{\left[u_{N}(t)-\int_{0}^{2 T} K\left(t, s, u_{N}(s)\right) d s-f(t)\right]_{t=\xi_{j i}}=0,} \\
i=1, \ldots, m ; \quad j=1, \ldots, 2 N .
\end{gathered}
$$

Theorem 3.2 (see [39, p. 143], cf. also [43], [42]). Let conditions (F1)-(F3) be fulfilled and let the collocation points (2.6) with gridpoints given by (3.4) be used.

Then there exist an $N_{0} \in \mathbb{N}$ and a real number $\delta_{0}>0$ such that for $N>N_{0}$, the collocation method (3.5) defines a unique approximation $u_{N} \in \tilde{E}_{N}$ to the solution $u_{0}$ of equation (3.1) satisfying $\left\|u_{N}-u_{0}\right\|_{L^{\infty}(0,2 T)} \leq \delta_{0}$. The following error estimates hold:

1) if $m<1-\nu$ then

$$
\max _{0 \leq t \leq 2 T}\left|u_{N}(t)-u_{0}(t)\right| \leq c h^{m} \text { for } r \geq 1 ;
$$

2) if $m=1-\nu$ then

$$
\max _{0 \leq t \leq 2 T}\left|u_{N}(t)-u_{0}(t)\right| \leq c \begin{cases}h^{m}(1+\log h \mid) & \text { for } r=1, \\ h^{m} & \text { for } r>1,\end{cases}
$$

and

$$
\left\|u_{N}-u_{0}\right\|_{L^{p}(0,2 T)} \leq c h^{m} \text { for } r \geq 1,1 \leq p<\infty ;
$$

3) if $m>1-\nu$ then

$$
\max _{0 \leq t \leq 2 T}\left|u_{N}(t)-u_{0}(t)\right| \leq c\left\{\begin{array}{lrr}
h^{r(1-\nu)} & \text { for } & 1 \leq r \leq \frac{m}{1-\nu}, \\
h^{m} & \text { for } & r \geq \frac{m}{1-\nu}
\end{array}\right.
$$

and

$$
\left\|u_{N}-u_{0}\right\|_{L^{P}(0,2 T)} \leq c \begin{cases}h^{r\left(1-\nu+\frac{1}{p}\right)} & \text { if } 1 \leq r<\frac{m}{1-\nu+\frac{1}{p}}, m>1-\nu+\frac{1}{p}, \\ h^{m}(1+|\log h|)^{\frac{1}{p}} & \text { if } r=\frac{m}{1-\nu+\frac{1}{p}}, m \geq 1-\nu+\frac{1}{p}, \\ h^{m} & \text { if } r>\frac{m}{1-\nu+\frac{1}{p}}, r \geq 1,\end{cases}
$$

for $1 \leq p<\infty$; 
4) if $r=r(m, \nu) \geq 1$ satisfies (2.4), then

$$
\tilde{\varepsilon}_{N} \leq c h^{m}
$$

where

$$
\tilde{\varepsilon}_{N}=\max _{i=1, \ldots, m ; j=1, \ldots, 2 N}\left|u_{N}\left(\xi_{j i}\right)-u_{0}\left(\xi_{j i}\right)\right| .
$$

Theorem 3.3 ([24]). Let the following conditions be fulfilled:

$\left(\mathrm{F}^{\prime}\right)$ The kernel $K(t, s, u)$ and $\partial K(t, s, u) / \partial u$ are $m+\mu+1$ times $(m, \mu \in \mathbf{Z}, m \geq$ $1,0 \leq \mu \leq m-1)$ continuously differentiable with respect to $t, s, u$ for $t, s \in[0,2 T], t \neq s, u \in \mathbb{R}$, and satisfy (2.2) and (2.3) with $i+j+k \leq$ $m+\mu+1,-\infty<\nu<1$.

(F2') $f \in C^{m+\mu+1, \nu}(0,2 T)$.

(F3) The integral equation (3.1) has a solution $u_{0} \in L^{\infty}(0,2 T)$, and the linearized equation (3.3) has in $L^{\infty}(0,2 T)$ only the trivial solution $v \equiv 0$.

(F4) The collocation points (2.6) are generated by the knots (2.7) of a quadrature formula (2.20) which is exact for all polynomials of degree $m+\mu, 0 \leq \mu \leq$ $m-1$.

(F5) The scaling parameter $r=r(m, \nu, \mu) \geq 1$ satisfies the conditions (2.18).

Then

$$
\tilde{\varepsilon}_{N} \leq \operatorname{ch}^{m} \begin{cases}h & \text { if } \nu<0, \\ h(1+|\log h|) & \text { if } \nu=0, \\ h^{1-\nu} & \text { if } \nu>0,\end{cases}
$$

where $\tilde{\varepsilon}_{N}$ is defined in (3.12).

If, in addition, we assume

(F6) $\nu<0, \mu \geq 1$, and for $0 \leq j \leq \min \{\mu-1,-\nu\}, 0 \leq k \leq \min \{\mu-1,-\nu\}$, the derivatives (2.23) are bounded and continuous on $[0,2 T] \times[0,2 T] \times[-\tau, \tau]$ with any $\tau>0$,

then

$$
\tilde{\varepsilon}_{N} \leq c h^{m} \begin{cases}h^{\mu+1} & \text { if } 1-\nu>\mu+1, \\ h^{\mu+1}(1+|\log h|) & \text { if } 1-\nu=\mu+1, \\ h^{1-\nu} & \text { if } 1-\nu<\mu+1 .\end{cases}
$$

\section{Proof of Theorems 2.1-2.4}

Assume that the kernel $K(t, s, u)$ of equation (2.1) satisfies (V1) for $0 \leq s<t \leq$ $T,-\infty<u<\infty$. Assume also that the forcing function $f(t)$ of equation (2.1) satisfies (V2) for $0<t \leq T$, and let $u_{0} \in L^{\infty}(0, T)$ be a solution of equation (2.1). First of all we extend $K(t, s, u)$ up to a $\tilde{K}(t, s, u)$ which will satisfy condition (F1) for $0 \leq t \leq 2 T, 0 \leq s \leq 2 T, t \neq s, u \in \mathbb{R}$.

We shall use an extension method based on reflecting (see, for example [17]): for a given function $v \in C^{m}[a-\tau, a], m \in \mathbb{N}, a, \tau \in \mathbb{R}, \tau>0$, put

$$
\tilde{v}(s)= \begin{cases}v(s) & \text { for } s \in[a-\tau, a], \\ \sum_{j=0}^{m} c_{j} v(a-j(s-a)) & \text { for } s \in\left(a, a+\frac{\tau}{m}\right],\end{cases}
$$


where

$$
\sum_{j=0}^{m}(-j)^{k} c_{j}=1, k=0,1 \ldots, m
$$

From (4.1) and (4.2) one obtains that

$$
\tilde{v} \in C^{m}\left[a-\tau, a+\frac{\tau}{m}\right]
$$

We now construct an extension for the kernel $K(t, s, u)$ of equation (1.1). First, for any $u \in \mathbb{R}, \frac{2}{3} T \leq t \leq T$, we extend $K(t, s, u)$ with respect to $s$ for $s<0$ (denoting this extension again by $K(t, s, u)$ ):

$$
K(t, s, u)=e(-s) \sum_{j=0}^{m} c_{j} K(t,-j s, u),-\frac{2 T}{3 m} \leq s<0 .
$$

Here $\left(c_{0}, c_{1}, \ldots, c_{m}\right)$ is the (unique) solution of the system (4.2) and $e \in C^{\infty}\left(\mathbb{R}_{+}\right)$ is a smooth function such that

$$
e(s)=\left\{\begin{array}{l}
1 \text { for } 0 \leq s \leq \frac{2 T}{9 m} \\
0 \text { for } \frac{2 T}{6 m} \leq s \leq T
\end{array}\right.
$$

Second, for any $u \in \mathbb{R}$, we extend $K(t, s, u)$ with respect to $t(T \leq t \leq 2 T)$ along the lines $s=t-\gamma(0<\gamma \leq 2 T)$ :

$$
K_{1}(t, s, u)=\left\{\begin{array}{rc}
K(t, s, u) & \text { for } 0 \leq s<t \leq T, \\
e(t-T) \sum_{j=0}^{m} c_{j} K(T-j(t-T), T-j(s+\gamma-T)-\gamma, u) & \text { for } T \leq s<t \leq 2 T, t-s=\gamma .
\end{array}\right.
$$

Finally, in the third step we put

$$
\tilde{K}(t, s, u)= \begin{cases}K_{1}(t, s, u) e_{1}(u) & \text { for } 0 \leq s<t \leq 2 T \\ 0 & \text { for } 0 \leq t<s \leq 2 T\end{cases}
$$

with an $e_{1} \in C_{0}^{\infty}(\mathbb{R}), e_{1}(u)=1$ for $|u| \leq 2 M, e_{1}(u)=0$ for $|u| \geq 3 M$, where $M=\max _{0 \leq t \leq T}\left|u_{0}(t)\right|$. It is easy to check that the function $\tilde{K}(t, s, u)$ satisfies (F1).

Indeed, using (V1) we obtain that $\tilde{K}(t, s, u)$ is $m$ times continuously differentiable with respect to $t, s, u$ for $t \in[0,2 T], s \in[0,2 T], t \neq s, u \in \mathbb{R}$. Furthermore, it follows from the above construction that:

1) if $0 \leq s<t \leq T, u \in \mathbb{R}$, then

$$
\begin{aligned}
\left(\frac{\partial}{\partial t}\right)^{i} & \left(\frac{\partial}{\partial t}+\frac{\partial}{\partial s}\right)^{j}\left(\frac{\partial}{\partial u}\right)^{k} \tilde{K}(t, s, u) \\
= & \left(\frac{\partial}{\partial t}\right)^{i}\left(\frac{\partial}{\partial t}+\frac{\partial}{\partial s}\right)^{j}\left(\frac{\partial}{\partial u}\right)^{k}\left[K(t, s, u) e_{1}(u)\right]
\end{aligned}
$$


2) if $T \leq s<t \leq 2 T, u \in \mathbb{R}$, then

$$
\begin{aligned}
&\left(\frac{\partial}{\partial t}\right)^{i}\left(\frac{\partial}{\partial t}+\right.\left.\frac{\partial}{\partial s}\right)^{j}\left(\frac{\partial}{\partial u}\right)^{k} \tilde{K}(t, s, u) \\
&=\left[\left(\frac{\partial}{\partial t}\right)^{i}\left(\frac{\partial}{\partial t}+\frac{\partial}{\partial s}\right)^{j}\left(\frac{\partial}{\partial u}\right)^{k} e(t-T)\right] \\
& \quad \times \sum_{p=0}^{m} c_{p} K(T-p(t), T-p(s+\gamma-T)-\gamma, u) e_{1}(u) \\
&+e(t-T) \sum_{p=0}^{m} c_{p}\left(\frac{\partial}{\partial t}\right)^{i}\left(\frac{\partial}{\partial t}+\frac{\partial}{\partial s}\right)^{j}\left(\frac{\partial}{\partial u}\right)^{k} \\
& \times\left[K(T-p(t-T), T-p(s+\gamma-T)-\gamma, u) e_{1}(u)\right]
\end{aligned}
$$

3) if $0 \leq t<s \leq 2 T$ then

$$
\left(\frac{\partial}{\partial t}\right)^{i}\left(\frac{\partial}{\partial t}+\frac{\partial}{\partial s}\right)^{j}\left(\frac{\partial}{\partial u}\right)^{k} \tilde{K}(t, s, u)=0 .
$$

In case 1) we obtain the estimates (2.2) and (2.3) for $\tilde{K}(t, s, u)$ using Assumption (V1). In case 3) statements (2.2) and (2.3) with respect to $\tilde{K}(t, s, u)$ are trivially fulfilled.

Now consider case 2). Using (4.3), (4.5) and (V1), we are led to

$$
\begin{gathered}
\left|\left(\frac{\partial}{\partial t}\right)^{i}\left(\frac{\partial}{\partial t}+\frac{\partial}{\partial s}\right)^{j}\left(\frac{\partial}{\partial u}\right)^{k} \tilde{K}(t, s, u)\right| \\
\leq c b_{1}(3 M)\left[\begin{array}{ll}
1 & \text { if } \nu<0 \\
1+|\log | t-s|| & \text { if } \nu=0 \\
|t-s|^{-\nu} & \text { if } \nu>0
\end{array}\right\}+\left\{\begin{array}{ll}
1 & \text { if } \nu+i<0 \\
1+|\log | t-s|| & \text { if } \nu+i=0 \\
|t-s|^{-\nu-i} & \text { if } \nu+i>0
\end{array}\right] \\
\leq c^{\prime} b_{1}(3 M) \begin{cases}1 & \text { if } \nu+i<0, \\
1+|\log | t-s|| & \text { if } \nu+i=0, \\
|t-s|^{-\nu-i} & \text { if } \nu+i>0 .\end{cases}
\end{gathered}
$$

In a similar way we verify that condition $(2.3)$ is also satisfied.

So, we have shown that the function $\tilde{K}(t, s, u)$ is $m$ times continously differentiable with respect to $t, s, u$ for $t, s, u \in[0,2 T], t \neq s, u \in \mathbb{R}$, and satisfies conditions (2.2) and (2.3) with some $b_{1}$ and $b_{2}$ independent of $|u|$ and $\max \left\{\left|u_{1}\right|,\left|u_{2}\right|\right\}$, respectively. Thus, $\tilde{K}(t, s, u)$ also satisfies the global Lipschitz condition: for $t \in[0,2 T]$, $s \in[0, t), u_{1}, u_{2} \in \mathbb{R}$,

$$
\left|\tilde{K}\left(t, s, u_{1}\right)-\tilde{K}\left(t, s, u_{2}\right)\right| \leq \tilde{b} \begin{cases}1 & \text { if } \nu<0, \\ 1+|\log | t-s|| & \text { if } \nu=0, \\ |t-s|^{-\nu} & \text { if } \nu>0\end{cases}
$$

with a constant $\tilde{b}$ not depending on $u_{1}$ and $u_{2}$. 
We now define

$$
\tilde{f}(t)= \begin{cases}f(t) & \text { for } 0<t \leq T, \\ e(t-T) \sum_{j=0}^{m} c_{j} f(T-j(t-T)) & \text { for } T<t \leq 2 T,\end{cases}
$$

where $\left(c_{0}, c_{1}, \ldots, c_{m}\right)$ is the solution of the system $(4.2)$ and $e \in C^{\infty}\left(\mathbb{R}_{+}\right)$is defined in (4.3). It is clear that $\tilde{f} \in C^{m, \nu}(0,2 T) \subset C[0,2 T]$.

Consider the equation

$$
u(t)=\int_{0}^{2 T} \tilde{K}(t, s, u(s)) d s+\tilde{f}(t), \quad 0 \leq t \leq 2 T,
$$

where $\tilde{K}$ and $\tilde{f}$ are defined by (4.4) and (4.7). Equation (4.8) is actually a Volterra integral equation, since $\tilde{K}(t, s, u)=0$ for $0 \leq t<s \leq 2 T$. Due to the weak singularity of the kernel $\tilde{K}(t, s, u)$ and the global Lipschitz condition (4.6), (4.8) is uniquely solvable in $C[0,2 T]$; let $\tilde{u}_{0} \in C[0,2 T]$ denote the solution. It follows from the above construction that $\tilde{u}_{0}(t)=u_{0}(t)$ for $0 \leq t \leq T$. According to Theorem 3.1, $\tilde{u}_{0} \in C^{m, \nu}(0,2 T)$; therefore $u_{0} \in C^{m, \nu}(0, T]$. Theorem 2.1 is thus proved.

Further, for $0 \leq t \leq 2 T$, the linear homogenous Fredholm equation

$$
v(t)=\int_{0}^{2 T} \tilde{K}_{0}(t, s) v(s) d s, \quad \tilde{K}_{0}(t, s)=\left.\frac{\partial \tilde{K}(t, s, u)}{\partial u}\right|_{u=\tilde{u}_{0}(s)},
$$

actually takes the form of a linear homogeneous Volterra equation,

$$
v(t)=\int_{0}^{t} \tilde{K}_{0}(t, s) v(s) d s
$$

and therefore has in $L^{\infty}(0,2 T)$ only the trivial solution $v \equiv 0$. Thus condition (F3) is fulfilled with respect to the equation (4.8). Finally, assume that the gridpoints (3.4) are used. Then the assertions of Theorem 2.2 follow immediately from the corresponding statements of Theorem 3.2 about equation (4.8).

Thus, Theorem 2.2 is proved.

In a similar way we obtain that the function $\tilde{K}(t, s, u)$ in (4.4) satisfies the conditions $\left(\mathrm{F} 1^{\prime}\right)$ and $(\mathrm{F} 6)$ whenever the kernel $K(t, s, u)$ of the equation (2.1) satisfies the conditions $\left(\mathrm{V}^{\prime}\right)$ and $(\mathrm{V} 6)$, respectively. Finally we obtain that the function $\tilde{f}(t)$ in (4.6) satisfies $\left(\mathrm{F}^{\prime}\right)$ as long as the forcing function $f(t)$ obeys $\left(\mathrm{V}^{\prime}\right)$, and condition (F3) will be fulfilled with respect to (4.8) as long as equation (2.1) is solvable in $L^{\infty}(0, T)$. Therefore, assuming that the conditions of Theorem 2.3 and Theorem 2.4, respectively, are fulfilled, the statements of Theorems 2.3 and 2.4 follow immediately from Theorem 3.3.

Hence, Theorems 2.3 and 2.4 are proved.

\section{Numerical example}

Let $m=2$, and let $\eta_{1}=-\frac{1}{\sqrt{3}}$ and $\eta_{2}=\frac{1}{\sqrt{3}}$ be the knots of the Gauss quadrature formula $\int_{-1}^{1} \varphi(\xi) d \xi \approx \varphi\left(\eta_{1}\right)+\varphi\left(\eta_{2}\right)$. In this case the approximate solution $u_{N} \in E_{N}$ to equation $(2.1)$ in the interval $\left[t_{j-1}, t_{j}\right](j=1, \ldots, N)$ can be represented in the form

$$
u_{N}(t)=c_{j 1} \frac{\xi_{j 2}-t}{\xi_{j 2}-\xi_{j 1}}+c_{j 2} \frac{t-\xi_{j 1}}{\xi_{j 2}-\xi_{j 1}}, \quad t_{j-1} \leq t \leq t_{j},
$$


where $\xi_{j 1}, \xi_{j 2}$ are the knots (2.6) and the coefficients $c_{j k}=u_{N}\left(\xi_{j k}\right)(k=1,2 ; j=$ $1, \ldots, N)$ are determined from the system $(2.10)(m=2)$. From Theorem 2.2 we obtain for the approximate solution (5.1) the estimate

$$
\varepsilon_{N} \leq c h^{2} \quad \text { for } \begin{cases}r>\frac{1}{1-\nu} & \text { if } \quad \nu \geq 0 \\ r \geq 1 & \text { if } \quad \nu<0\end{cases}
$$

where $h=T / N$ and

$$
\varepsilon_{N}=\max _{i=1,2 ; j=1, \ldots, N}\left|u_{N}\left(\xi_{j i}\right)-u_{0}\left(\xi_{j i}\right)\right| .
$$

From Theorems 2.3 and 2.4, we obtain the following estimates for the error (5.3) (see $(2.22),(2.24), m=2$ with $\mu=1$ ):

$$
\varepsilon_{N} \leq \operatorname{ch}^{2} \begin{cases}h & \text { if } \nu<0 \\ h(1+|\log h|) & \text { if } \nu=0 \\ h^{1-\nu} & \text { if } \nu>0\end{cases}
$$

or (under the conditions of Theorem 2.4)

$$
\varepsilon \leq c h^{2} \begin{cases}h^{2} & \text { if } \nu<-1 \\ h^{2}(1+|\log h|) & \text { if } \nu=-1 \\ h^{1-\nu} & \text { if } \nu>-1\end{cases}
$$

provided that

$$
\begin{cases}r \geq 1 & \text { if } \quad \nu<-2 \\ r>\frac{4}{2-\nu} & \text { if }-2 \leq \nu \leq-1 \\ r \geq \frac{3-\nu}{2-\nu} & \text { if }-1<\nu<1-\sqrt{2} \\ r>\frac{2}{1-\nu} & \text { if } 1-\sqrt{2} \leq \nu<1\end{cases}
$$

Now we present the following example. Consider the integral equation

$$
u(t)=\int_{0}^{t}(t-s)^{-1 / 2} u^{2}(s) d s+t^{1 / 2}\left(1-\frac{4}{3} t\right), \quad 0 \leq t \leq T .
$$

It is easy check that $u_{0}(t)=t^{1 / 2}$ is the exact solution to equation (5.7) and Assumptions $\left(\mathrm{V}^{\prime}\right)$ and $\left(\mathrm{V}^{\prime}\right)$ of Theorem 2.3 are fulfilled with $\nu=1 / 2, m=2$, $\mu=1$.

The equation (5.7) was solved numerically by the collocation method (2.10) $(m=2)$, where the Gauss points $\eta_{2}=-\eta_{1}=1 / \sqrt{3}$ were used for determining the collocation points (2.6), and we chose $r=41 / 10>2 /(1-\nu)$ (see (5.6)). At every step (on every subinterval $\left.\left[t_{j-1}, t_{j}\right]\right)$ the coefficients $c_{j i}=u_{N}\left(\xi_{j i}\right)(i=1,2)$ were calculated from $(2.10)(m=2)$ by the Newton method. All the integrals which are needed for the construction of the system (2.10) were found analytically. Some of the numerical results for $T=3 / 4$ are presented in the following Table 1 , where $\varepsilon_{N}$ is defined in (5.3). The experiments were carried out on an IBM 4381 (in double precision).

From Table 1 we can see that the numerical results are consistent with the theoretical estimate, which is $\varepsilon_{N}=O\left(h^{5 / 2}\right), h=0.75 / N$. Notice that the number of collocation points (the number of unknowns) is $2 N$. 


\section{TABLE 1}

\begin{tabular}{rcc}
\hline \multicolumn{1}{c}{$N$} & $\varepsilon_{N}(r=41 / 10)$ & $\varepsilon_{N / 2} / 2^{5 / 2}$ \\
\hline 4 & $0.55 \mathrm{E}-01$ & \\
8 & $0.80 \mathrm{E}-02$ & $0.97 \mathrm{E}-02$ \\
16 & $0.12 \mathrm{E}-02$ & $0.14 \mathrm{E}-02$ \\
32 & $0.19 \mathrm{E}-03$ & $0.21 \mathrm{E}-03$ \\
64 & $0.32 \mathrm{E}-04$ & $0.33 \mathrm{E}-04$ \\
128 & $0.20 \mathrm{E}-05$ & $0.57 \mathrm{E}-05$ \\
\hline
\end{tabular}

In the same example on longer intervals (e.g. $T=4$ ), some instability of the approximate solution was observed. The numerical stability of the scheme is worth examining independently.

\section{ACKNowledgments}

The research of the first author was supported by the Natural Sciences and Engineering Research Council of Canada (Research Grant Nr. OGP0009406). The research of the second author was supported by the Estonian Science Foundation (Research Grant Nr. 2999).

\section{REFERENCES}

1. C. de Boor, A Practical Guide to Splines, Springer-Verlag, New York, 1978. MR 80a:65027

2. H. Brunner, Nonpolynomial spline collocation for Volterra equations with weakly singular kernels, SIAM J. Numer. Anal., 20 (1983), 1106-1119. MR 85d:65069

3. H. Brunner, The numerical solution of weakly singular Volterra integral equations by collocation on graded meshes, Math. Comp. 45 (1985), 417-437. MR 87b:65223

4. H. Brunner, The approximate solution of Volterra equations with nonsmooth solutions, Utilitas Math., 27 (1985), 57-95. MR 87b:45027

5. H. Brunner, Collocation methods for one-dimensional Fredholm and Volterra integral equations, in The State of the Art in Numerical Analysis (A. Iserles and M.J.D. Powell, eds.), Clarendon Press, Oxford, 1987: pp. 563-600. MR 89m:65112

6. H. Brunner, and P. J. van der Houwen, The Numerical Solution of Volterra Equations, NorthHolland, Amsterdam, 1986. MR 88g:65136

7. G. A. Chandler, Superconvergence of numerical methods to second kind integral equations. PhD Thesis, 1979, Australian National University, Canberra.

8. I. G. Graham, Singularity expansions for the solutions of second kind Fredholm integral equations with weakly singular convolution kernels, J. Integral Equations, 4 (1982), 1-30. MR 83e: 45006

9. I. G. Graham, Galerkin methods for second kind integral equations with singularities. Math. Comp., 1982, 39, 519-533. MR 84d:65090

10. W. Hackbusch, Integralgleichungen. Theorie und Numerik, Teubner, Stuttgart, 1989. MR 90g: 45001

11. F. de Hoog and R. Weiss, On the solution of a Volterra integral equation with a weakly singular kernel, SIAM J. Math Anal., 4 (1973), 561-573. MR 49:1818

12. H. Kaneko, R. Noren and Y. Xu, Regularity of the solution of Hammerstein equations with weakly singular kernel, Integral Equations Operator Theory, 13 (1990), 660-670. MR 92a: 45002

13. H. Kaneko, R. Noren and Y. Xu, Numerical solutions for weakly singular Hammerstein equations and their superconvergence, J. Integral Equations Appl. 4, (1992), 391-406. MR 93j:65223

14. R. Kangro, On the smoothness of solutions to an integral equation with a kernel having a singularity on a curve, Acta et comm. Univ. Tartuensis 913 (1990), 24-37. MR 92e:45002 
15. U. Kangro, The smoothness of the solution of a two- dimensional integral equation with logarithmic kernel. Proc. Eston. Acad. Sci., Phys., Math 39 (1990), 196-204 (in Russian). MR 92a: 45012

16. U. Kangro, The smoothness of the solution to a two-dimensional integral equation with logarithmic kernel, Z. Anal. Anwendungen, 12 (1993), 305-318. MR 94i:45010

17. J. L. Lions and E. Magenes, Non-Homogeneous Boundary Value Problems and Applications, Vol. I, Springer-Verlag, Berlin, 1972. MR 50:2670

18. J. E. Logan, The approximate solution of Volterra integral equations of the second kind, $\mathrm{PhD}$ Thesis, 1976, University of Iowa, Iowa City.

19. Ch. Lubich, Runge-Kutta theory for Volterra and Abel integral equations of the second kind, Math. Comp., 41 (1983), 87-102. MR 85a:65178

20. R. K. Miller and A. Feldstein, Smoothness of solutions of Volterra integral equations with weakly singular kernels, SIAM J. Math. Anal., 2 (1971), 242-258. MR 44:4465

21. A. Pedas, On the solution of integral equations with logarithmic kernel by the first kind spline collocation method, Tartu Riiklik Ülikool, Toimetised (Acta et comm. Univ. Tartuensis), 431 (1977), 130-146 (in Russian). MR 58:3589

22. A. Pedas, On the smoothness of the solution of an integral equation with a weakly singular kernel, Tartu Riiklik Ülikool, Toimetised (Acta et comm. Univ. Tartuensis) 492 (1979), 56-68 (in Russian). MR 82b:45010

23. A. Pedas and G. Vainikko, The smoothness of solutions to nonlinear weakly singular equations, Z. Anal. Anwendungen, 13 (1994), 463-476. MR 95e:45002

24. A. Pedas and G. Vainikko, Superconvergence of piecewise polynomial collocations for nonlinear weakly singular integral equations, J. Integral Equations Appl. 9 (1997), 379-406. CMP 98:11

25. J. Pitkäranta, On the differential properties of solutions to Fredholm equations with weakly singular kernels, J. Inst. Math. Phys., 1979, 24, 109-119. MR 80i:65157

26. J. Pitkäranta, Estimates for derivatives of solutions to weakly singular Fredholm integral equations, SIAM J. Math. Anal., 1980, 11, 952-968. MR 81m:45006

27. J. R. Rice, On the degree of convergence of nonlinear spline approximation, in: Approximation with Special Emphasis on Spline Functions (I. J. Schoenberg, ed.), Academic Press, New York, 1969: pp. 349-365. MR 42:2226

28. G. R. Richter, On weakly singular integral equations with displacement kernels, J. Math. Anal. Appl., 55 (1976), 32-42. MR 53:11322

29. H. J. J. te Riele, Collocation methods for weakly singular second-kind Volterra integral equations with non-smooth solution, IMA J. Numer. Anal., 2 (1982), 437-449. MR 84g:65167

30. C. Schneider, Regularity of the solution to a class of weakly singular Fredholm integral equations of the second kind, Integral Equations Operator Theory, 2 (1979), 62-68. MR 80f:45002

31. C. Schneider, Product integration for weakly singular integral equations, Math. Comp., 36, (1981), 207-213. MR 82c:65090

32. L. L. Schumaker, Spline Functions: Basic Theory, Wiley, New York, 1981. MR 82j:41001

33. E. Tamme, Two-grid methods for nonlinear multidimensional weakly singular integral equations, J. Integral Equations Appl., 7, (1995), 99-113. MR 96e:65080

34. P. Uba, The smoothness of solution of weakly singular integral equations with a discontinuous coefficient. Proc. Eston. Acad. Sci. Phys., Math. 37, No.2 (1988), 192-203 (in Russian). MR 89g:45004

35. P. Uba, A collocation method with cubic splines to the solution of a multidimensional weakly singular integral equation. Acta et comm. Univ. Tartuensis 863 (1989), 19-25. MR 91g:65315

36. P. Uba, A collocation method with cubic splines for multidimensional weakly singular nonlinear integral equations, J. Integral Equations Appl., 6 (1994), 257-266. MR 95g:45013

37. G. Vainikko, Piecewise polynomial approximation of a solution to multidimensional weakly singular integral equation. Acta et comm. Univ. Tartuensis 833 (1988), 19-26 (in Russian). MR 90i:65250

38. G. Vainikko, Collocation methods for multidimensional weakly singular integral equations. In: Numer. Anal. and Math. Modelling. Banach Center Publ.: Warsaw, 1990, 91-105 (in Russian). MR 92a:65355

39. G. Vainikko, On the smoothness of the solution of multidimensional weakly singular integral equations. Math. USSR Sbornik 68 (1991), 585-600. (Russian original 1989). MR 91a:45010

40. G. Vainikko, Multidimensional Weakly Singular Integral Equations, Lecture Notes Math., Vol. 1549, Springer-Verlag, Berlin-Heidelberg-New York, 1993. MR 94i:45001 
41. G. Vainikko and A. Pedas, The properties of solutions of weakly singular integral equations, J. Austral. Math. Soc. Ser. B, 22 (1981) 419-430 MR 82i:45014

42. G. Vainikko, A. Pedas, P. Uba Methods for Solving Weakly Singular Integral Equations, Univ. of Tartu, Tartu, 1984 (in Russian).

43. G. Vainikko and P. Uba, A piecewise polynomial approximation to the solution of an integral equation with weakly singular kernel, J. Austral. Math. Soc. Ser. B, 22 (1981), 431-438.

Department of Mathematics and Statistics, Memorial University of Newfoundland, St. John's, Nfld., Canada A1C 5S7

E-mail address: hermann@math.mun.ca

Department of Applied Mathematics, University of Tartu, 0000 Litvi 2-206, Tartu EE2400, Estonia

E-mail address: Arvet.Pedas@ut.ee

Institute of Mathematics, Helsinki University of Technology, P.O.Box 1100, FiN02015 HUT, FinLAND

E-mail address: Gennadi.Vainikko@hut.fi 